Hydrol. Earth Syst. Sci., 10, 233-243, 2006

www.hydrol-earth-syst-sci.net/10/233/2006/

(C) Author(s) 2006. This work is licensed

under a Creative Commons License.

\title{
On the asymptotic behavior of flood peak distributions
}

\author{
E. Gaume \\ Centre d'Enseignement et de Recherche Eau Ville Environnement, Ecole Nationale des Ponts et Chaussées, Paris, France
}

Received: 2 August 2005 - Published in Hydrol. Earth Syst. Sci. Discuss.: 1 September 2005

Revised: 22 November 2005 - Accepted: 22 February 2006 - Published: 26 April 2006

\begin{abstract}
This paper presents some analytical results and numerical illustrations on the asymptotic properties of flood peak distributions obtained through derived flood frequency approaches. It confirms and extends the results of previous works: i.e. the shape of the flood peak distributions are asymptotically controlled by the rainfall statistical properties, given limited and reasonable assumptions concerning the rainfall-runoff process. This result is partial so far: the impact of the rainfall spatial heterogeneity has not been studied for instance. From a practical point of view, it provides a general framework for analysis of the outcomes of previous works based on derived flood frequency approaches and leads to some proposals for the estimation of very large return-period flood quantiles. This paper, focussed on asymptotic distribution properties, does not propose any new approach for the extrapolation of flood frequency distribution to estimate intermediate return period flood quantiles. Nevertheless, the large distance between frequent flood peak values and the asymptotic values as well as the simulations conducted in this paper help quantifying the ill condition of the problem of flood frequency distribution extrapolation: it illustrates how large the range of possibilities for the shapes of flood peak distributions is.
\end{abstract}

\section{Introduction}

Eagleson (1972) was the first to combine a rainfall stochastic model and a rainfall runoff model to generate synthetic "derived flood frequency distributions" (DFFD). The objective of this approach was twofold. Firstly, it aimed at understanding the relationship between the flood peak distributions (FPD) and the climatic and hydrologic characteristics of a watershed: to identify the main control parameters of

Correspondence to: E. Gaume

(gaume@cereve.enpc.fr) the FPD shape, to compare the FPD of various watersheds or to anticipate the effect of changes, for instance land use or climatic evolutions on FPD. Secondly, it appeared intellectually more satisfactory, for statistical interpolation and extrapolation purposes, to derive the shape of flood peak distributions from the selection of a rainfall statistical model and of a rainfall-runoff model adapted to the considered case study, rather than to directly select a theoretical distribution chosen on the basis of the extreme value theory or for mathematical convenience.

As statistical extrapolation tools, DFFD, if properly used, probably have performances comparable to the conventional procedure based on theoretical distribution fitting. But there is, for the moment, no reason to think that they perform better. The unavoidable simplifications of the runoff generating processes and of the rainfall statistical structure in the DFFD tools, the limited extrapolation capacities of the available simplified rainfall-runoff models reduce their potential advantage over conventional statistical extrapolation methods as illustrated by some works (Raines and Valdes, 1993; Moughamian et al., 1987). This probably explains why despite the numerous works conducted on DFFD since the first paper of Eagleson (De Michele and Salvadori, 2002; Loukas, 2002; Arnaud and Lavabre, 2002; Blazkova and Beven, 2002; Goel et al., 2000; Cameron et al., 2000; Iacobellis and Fiorentino, 2000; Gupta et al., 1996; Raines and Valdes, 1993; Smith, 1992; Sivapalan et al., 1990), such procedures are, to our knowledge, seldom used in an operational context (Lamb and Kay, 2004; Blazkova and Beven, 2004; Arnaud and Lavabre, 2000).

Nevertheless, DFFD are also interesting tools to study the functional relationship between the FPD shape and the climatic and hydrologic characteristics of the corresponding watershed. But what general conclusions about the shape of the FPD can be drawn on the basis of DFFD approaches? This question is still wide open. A large variety of rainfall stochastic models and rainfall-runoff models have been

Published by Copernicus GmbH on behalf of the European Geosciences Union. 
tested in the previous works on DFFD. Some of these works are purely numerical approaches based on Monte-Carlo simulations (Loukas, 2002; Arnaud and Lavabre, 2002; Hashemi et al., 2000). With an ad hoc choice of rainfall and rainfallrunoff models it is sometimes possible to derive an approximate (Diaz-Granados et al., 1984; Eagleson, 1972) or a completely analytical form of the resulting FPD (De Michele and Salvadori, 2002; Goel et al., 2000). The impact of the parameters of the rainfall and the rainfall-runoff models used on the FPD shape are generally analyzed but it is not possible through these various works to evaluate the influence of the models themselves.

The shape of a FPD, as will be shown hereafter, highly depends of course on the dynamics of the rainfall-runoff processes and the range of possibilities is quite large. But, general conclusions can be drawn concerning the asymptotic behavior of the FPD (i.e. the shape of the FPD as the return period or the peak discharge tends to infinity) for a large variety of rainfall-runoff dynamics and DFFD tools.

This paper explores the link between the rainfall intensity statistical characteristics and the asymptotic behavior of FPDs. The results presented are a generalization of results already obtained on specific DFFD tools (De Michele and Salvadori, 2002; Eagleson, 1972). Limited and reasonable assumptions are made concerning the rainfall-runoff process. The demonstration is first conducted with a simple rainfall stochastic model used in many previous DFFD works: the rainfall events are supposed to be rectangular pulses with a given duration and a constant intensity. Two asymptotic rainfall intensity distribution types are considered: extreme value distribution of type I (EV I) also called exponential type and EV II (hyper-exponential type).

The results are then generalized to any rainfall temporal structure. Numerical results obtained with a DFFD tool combining a 5-min point rainfall stochastic model (Mouhous et al., 2001) and a rainfall-runoff model presented in Appendix A1 are shown as an illustration.

The last part of the paper is devoted to the discussion. The "Gradex" statistical extrapolation method based on assumptions concerning the asymptotic behavior of flood peak distributions as well as the limits of the common practice consisting of fitting theoretical statistical distributions to short series of peak discharges are questioned in light of the results presented herein.

This work remains partial so far: the influence of the spatial heterogeneity of rainfall is for instance not studied.

\section{Basic concept}

2.1 The key idea: the simplification of the rainfall-runoff process in a DFFD framework

Let us begin with a very simple representation of a rainfall event before generalizing: a rectangular pulse (i.e. a constant intensity event over a given duration). This is the representation selected in many papers dealing with DFFD, including the paper of Eagleson (1972).

In this very simple case, any rainfall-runoff model can be summarized in the following form, as far as the peak discharge is concerned:

$Y=C X$

The peak discharge $Y$ is a given proportion of the rainfall intensity $X$. If the baseflow is neglected, this proportion $C$, a kind of runoff rate, is included in the interval $[0,1]$ and may depend on the duration of the rainfall event, the rainfallrunoff dynamics and the state of the watershed (antecedent soil moisture for instance). Note that the condition imposed on $C$ implies reasonable conditions concerning the rainfallrunoff process: the peak discharge can not be a negative value and can not exceed the intensity of the rainfall event. If $p(x)$ is the probability density function of the rainfall intensity $X$, then the survival function of $Y$ has the following form:

$P(Y \geq y)=F(y)=\int_{0}^{1} \int_{y / c}^{\infty} p(c \mid x) p(x) d x d c$

where $p(c \mid x)$ is the conditional density of $C$ given $X$. The survival function of $Y$ depends of course on the function $p(c \mid x)$ (i.e. on the rainfall-runoff dynamics), but its asymptote when $y$ tends to infinity is controlled by the distribution of $X$ as will be shown hereafter.

\subsection{The solution when $C$ is independent of $X$}

When $C$ is independent of $X$, then it is straightforward to demonstrate that the asymptotic distributions of $X$ and $Y$ belong to the same extreme value (EV) type and have the same shape parameter. If $C$ is not equal to zero and if the distribution of $X$ has no upper bound, than the distribution of $Y$ has no upper bound either: its asymptote is necessarily of the EV I (exponential) or EV II (hyper-exponential or algebric) type. Moreover recalling that the moments of the product of two independent random variables is equal to the product of their moments, if $X$ has an exponential asymptotic distribution (i.e. all the moments of $X$ are finite), than all the moments of $Y$ will also exist (i.e. the asymptotic distribution of $Y$ is of the exponential type). Conversely, if the asymptotic distribution of $X$ is of the EV II type, the moments of $X$ of orders greater than $\alpha$ (shape parameter of the asymptotic distribution) will be infinite. The moments of order greater than $\alpha$ of the random variable $Y$ will also be infinite. The asymptotic distribution of $Y$ is therefore also of the EV II type and has furthemore the same shape parameter $\alpha$ as the asymptotic distribution of $X$. The same results can be obtained deriving the asymptotic properties of the function $P(Y \geq y)$ see Appendix B1 and Appendix D1. It is furthermore demonstrated in Appendix B1 that if the density function of $C$ is not equal to zero for $c=1$, than the asymptotic distributions 

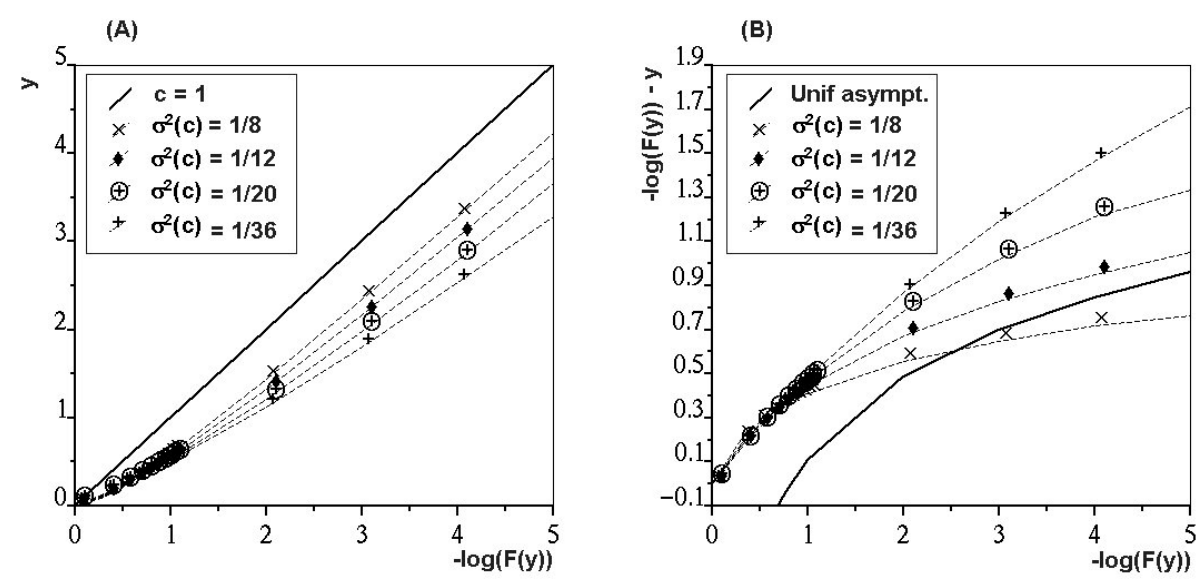

Fig. 1. Distributions of $Y=C X$ obtained with various values of the variance of $C$. The mean of $C$ is equal to 0.5 . The black diamonds correspond to a uniform distribution for $C$ over $[0,1]$.

(A)

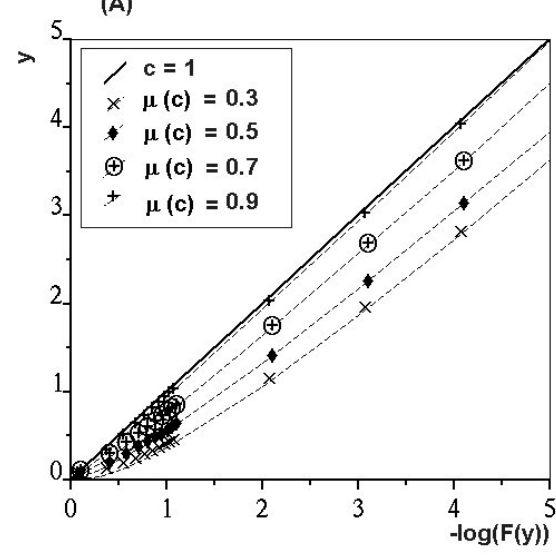

(B)

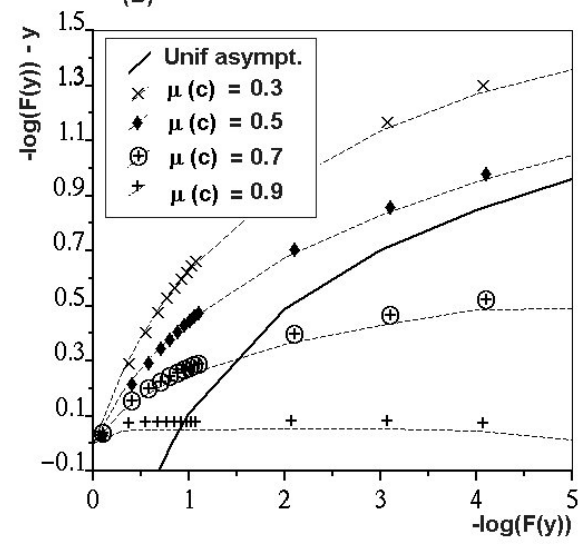

Fig. 2. Distributions of $Y=C X$ obtained with various values of the mean of $C$. The variance of $C$ is equal to $1 / 12$. The black diamonds correspond to a uniform distribution for $C$ over $[0,1]$.

of $X$ and $Y$ have the same scale parameter in the EV I case: both survival functions will be parallel to one another on a semi-logarithmic (Gumbel) plot.

Figures 1 and 2 show the distributions of $Y=C X$ obtained through Monte Carlo simulations with $X$ exponentially distributed (mean equal to 1 ) and $C$ being a Beta random variable taking values in the interval $[0,1]$. Different values for the mean and the variance of this Beta variable have been tested. In each case, the slope of the distribution of $Y$ appears to converge, even though very slowly, towards 1: the slope of the distribution of $X$ (continuous lines in Figs. 1a and $2 \mathrm{~b}$ ). It may appear more clearly on Figs. $1 \mathrm{~b}$ and $2 \mathrm{~b}$ that the slope of the distribution of $-\log F(y)-y$ tends towards 0 as $-\log F(y)$ or $y$ tend to infinity. The asymptote when $C$ is uniformly distributed is $-\log F(y)-y=\log (y)$ (see Appendix C1).

\section{Generalization of the previous results}

3.1 Rainfall events still have a constant intensity but the density function of $C$ may depend on $X$

The statistical independence between $C$ and $X$, that is the independence between the rainfall intensity and the "runoff rate" is an unrealistic assumption if the model is supposed to simulate a runoff process. In the case of an "infiltration excess" also called "Horton" runoff process, $C$ and $X$ are clearly linked. If a "saturation excess" process is simulated, $C$ is related to the rainfall amount of each event. The evolution of the density $p(c \mid x)$ with $x$ will, in this last case, depend on the relation between the intensity and the rainfall amount of an event. Since rectangular pulse events are considered, it will depend on the relation between the intensity and the duration of the rainfall events. The conditional expectancy of the rainfall volume of an event may decrease as its intensity 


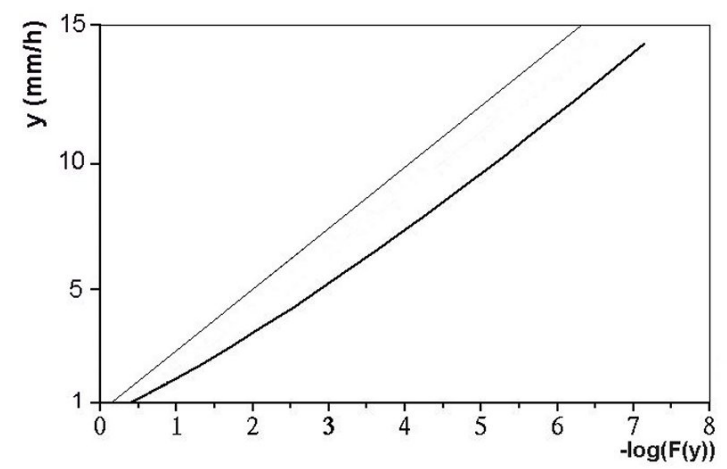

Diaz-Granados et al. (1984) : $100 \mathrm{~km}^{2}$

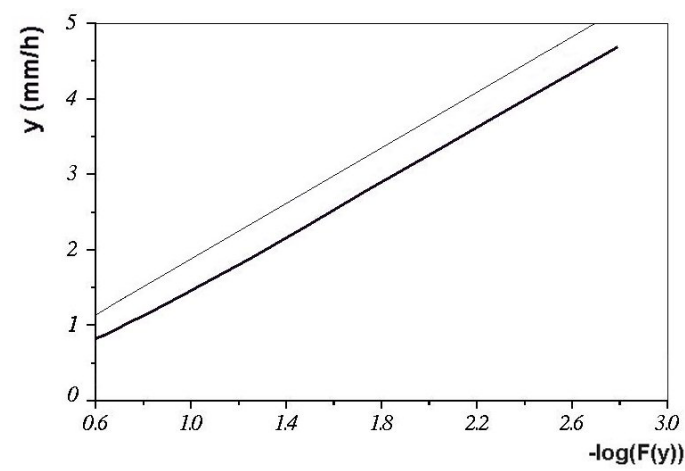

Eagleson (1972) : $300 \mathrm{~km}^{2}$

Fig. 3. Example of peak specific discharge frequency distributions obtained with two DFFD models (thick lines) and comparison with the distributions of the rainfall event intensities (thin lines).

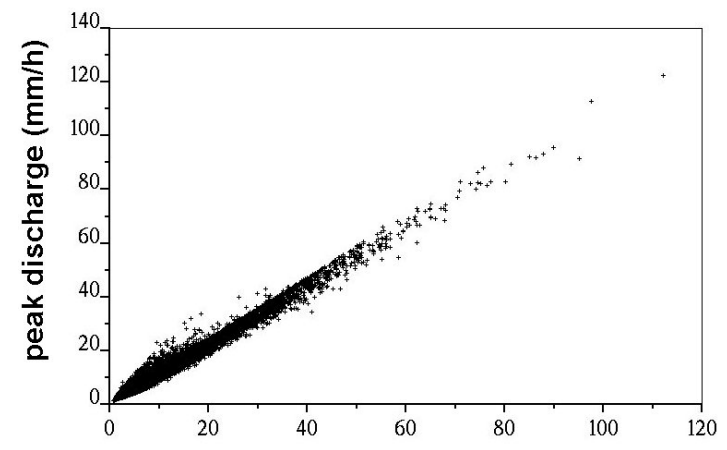

1-hour maximum rainfall intensity $(\mathrm{mm} / \mathrm{h})$

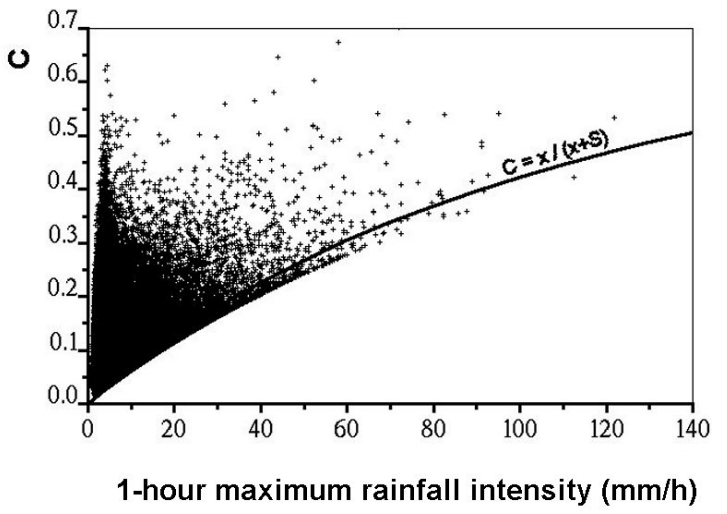

1-hour maximum rainfall intensity $(\mathrm{mm} / \mathrm{h})$

Fig. 4. Relations between the simulated peak discharge $(\mathrm{CN}=100)$, the simulated runoff rate $\mathrm{C}(\mathrm{CN}=65)$ and the rainfall event 1 -h maximum rainfall intensity. Linear transfer model.

increases in the very tricky situation where the rainfall event duration and intensity are highly negatively correlated (case tested by Goel et al., 2000). But usually, even if the rainfall event intensity and duration are slightly negatively correlated, the expectancy of the rainfall volume and therefore, the expectancy of the "runoff rate" will increase as the rainfall intensity of an event increases. Moreover, the event intensity distribution seems to control the asymptotic shape of the volume distribution (De Michele and Salvadori, 2003). It can therefore be foreseen, for "realistic" rainfall stochastic model and rainfall-runoff model combinations, that the runoff rate expectancy will have a general tendency to grow as the magnitude of the intensity of the rainfall event grows.

The function $p(c \mid x)$ can have two types of behaviors as $x$ tends to infinity. It can either converge towards a limit density function $p^{*}(c)$ defined over an interval $\left[c_{1}, c_{2}\right]$ with $c_{1}<c_{2}$ or it can concentrate around one value $c_{2}$. In this last case, $Y$ is asymptotically equal to $c_{2} X$ : i.e. the asymptotic distribution of the flood peak discharges is the distribution of the rainfall intensities multiplied by $c_{2}$. Let us add that if $c=1$ belongs to the domain of the possible, which is the case for most hydrological models if the rainfall spatial heterogeneity is not considered, than $c_{2}=1$. In other words, the asymptotic statistical distribution of the peak discharges of a watershed obtained through a DFFD framework will either have the same shape parameter, and scale parameter in the EV I case, than the distribution of the rainfall event intensities or be the distribution of the rainfall intensities.

This result confirms and extends the conclusions drawn from the detailed analysis of some specific DFFD frameworks: i.e. for DFFD frameworks in which rainfall events are considered as rectangular pulses, asymptotically "the shape parameter of the flood distribution is the same as that of the rainfall (intensity) distribution" (De Michele and Salvadori, 2002). Figure 3 illustrates this for two previous DFFD tools. Note that in the study of Eagleson, the runoff is supposed to be produced on a part of the total catchment area: the direct runoff producing area. The specific peak discharges have been computed considering this direct runoff producing area. 

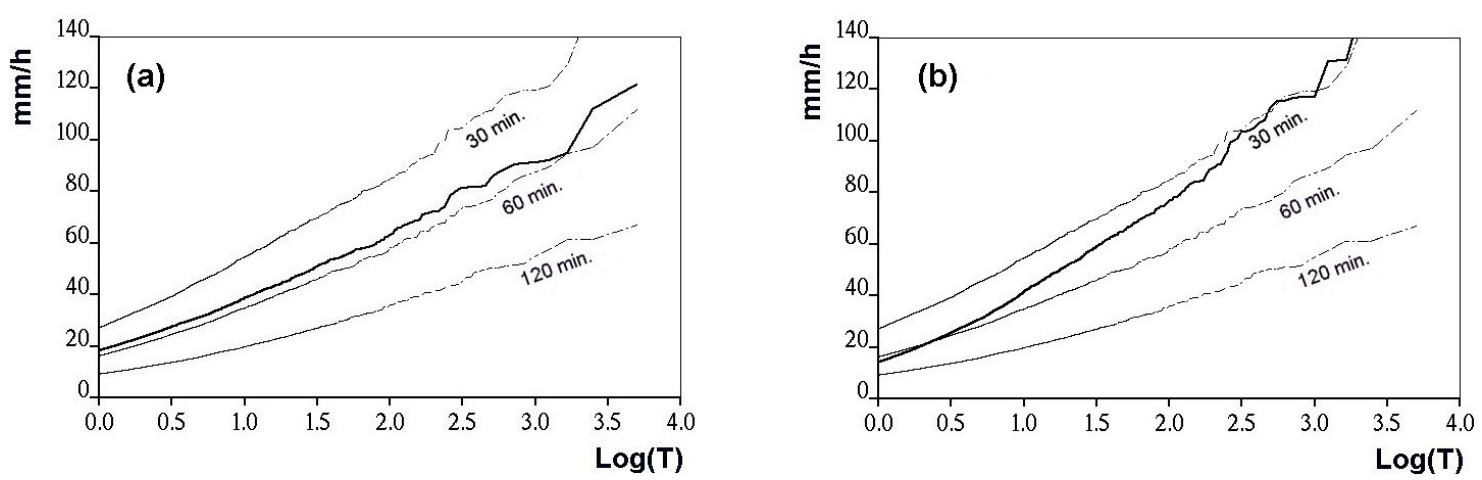

Fig. 5. Simulated flood peak $(\mathrm{mm} / \mathrm{h})$ of a theoretical impervious watershed (bold line) compared to the rainfall event maximum mean intensities over various durations (other lines): (a) linear tranfer and (b) kinematic wave.

\subsection{Generalization to any type of rainfall representation}

In the more general case, where the temporal variations of rainfall intensities during an event are considered, the peak discharge expressed in $\mathrm{mm} / \mathrm{h}$ can be higher than the mean intensity of the event. The coefficient $C$ in Eq. (1) is not limited to 1 any more. But a formula, comparable to Eq. (1) can be proposed to summarize the rainfall-peak discharge relation.

$Y=C Y_{m}$

where $Y_{m}$ represents the peak discharge of the watershed obtained for a runoff coefficient equal to 1: i.e. if the watershed is supposed to be impervious. It is the maximum possible peak discharge of a watershed. Again, in Eq. (3), the coefficient $C$ is included in the interval $[0,1]$ if the baseflow is not considered. Let us note that there is a link between the distribution of $Y_{m}$ and the statistical characteristics of the rainfall and particularly the so-called intensity-durationfrequency curves. This relation depends on the transfer function of the flood flows on the watershed.

If this transfer function is linear, the maximum possible peak discharge is highly correlated with the maximum mean rainfall intensity over a duration generally lower than the time of concentration of the watershed (see Fig. 4a for an example on a theoretical watershed having a time of concentration of one hour). Hence, the statistical distribution of $Y_{m}$ is the distribution of the maximum mean rainfall intensities over this duration (Fig. 5a). Just recall that the high correlation between peak discharge values and mean rainfall intensity over a given duration when a constant runoff coefficient is used is at the basis of the development of the well known rational method.

If the transfer function is not linear, the link between the distribution of $Y_{m}$ and the Intensity-duration-frequency curves is less direct (Fig. 5b). When the "kinematic wave" model is used, the time of concentration of the watershed can decrease as the discharge and the water mean velocity increases. For the chosen theoretical watershed and parameters used in the computations presented in Fig. 5b, this time of concentration appears to be about $1 \mathrm{~h}$. for a discharge equal to $20 \mathrm{~mm} / \mathrm{h}$ and $30 \mathrm{~min}$. for a discharge equal to $120 \mathrm{~mm} / \mathrm{h}$. Looking at Fig. 5b, it appears that the maximum mean intensity of the rainfall event over a duration close to the time of concentration of the watershed still controls the shape of the distribution of $Y_{m}$ in the non-linear transfer case. But then, the time of concentration depends on the discharge or the return period.

Recalling the theoretical results of the first part of this paper, the flood peak discharge distribution obtained with any rainfall-runoff model will asymptotically either (1) have the same shape parameter, and scale parameter in the EV I case, than the distribution of $c_{2} Y_{m}$ if the density function of the coefficient $C$ tends to a dense function on the interval $\left[c_{1}, c_{2}\right]$, or (2) be the distribution of $c_{2} Y_{m}$ if the density function of $C$ concentrates around $c_{2}$ as $Y_{m}$ tends to infinity.

\section{Analysis of some simulation results}

Numerical simulations were conducted to illustrate the conclusions of the previous parts of this paper using a DFFD tool combining a 5-min. point rainfall stochastic model (Mouhous et al., 2001) and a rainfall-runoff model presented in Appendix A1. This rainfall-runoff model is simple and includes the so-called SCS-CN runoff production model and either a linear or a kinematic wave transfer function. The chosen theoretical watershed has a time of concentration equal to one hour when the linear tranfer function is used (see the high correlation between the peak discharge and the 100\% runoff model in Figs. 4a, 5a). Moreover, the expectancy of the ratio between the simulated peak discharge and the 1-h maximum intensity previously noted $C$ appears to increase as the 1-h intensity increases due to the selected runoff production model (Fig. 4b). Its minimum possible value is close to the minimum runoff rate simulated by the SCS-CN model before the transfer function is applied (bold line on Fig. 4b). Due to the properties of the SCS-CN model, $C$ converges towards 1 as the 1-h maximum intensity of an 

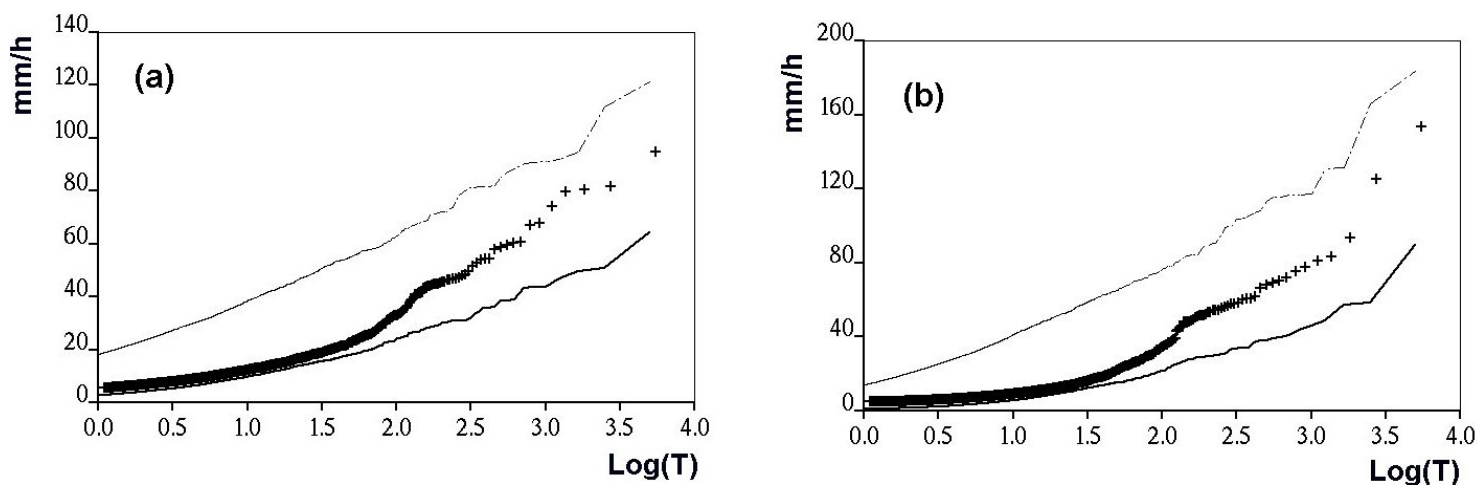

Fig. 6. Simulated flood peak ( $\mathrm{mm} / \mathrm{h}$ ) distributions for $\mathrm{CN}=100$ (thin line), $\mathrm{CN}=65$ (bold line), $\mathrm{CN}=65$ and modified $\mathrm{SCS}$ (dots): (a) linear tranfer and (b) kinematic wave.

event and hence as $Y_{m}$ (maximum peak discharge obtained when the watershed is impervious, i.e. $\mathrm{CN}=100$ ) tends towards infinity. According to the conclusions of the preceding parts of this paper, the asymptotic distribution of the simulated peak discharge should therefore be the distribution of $Y_{m}$. The simulation results (Fig. 6) indicate nevertheless that this convergence is very slow when the standard SCS$\mathrm{CN}$ model is used. Hydrological models simulating infiltration or saturation excess runoff generating processes will all show an increase of the runoff coefficient expectancy with the mean rainfall intensity over a given duration. But, the hydrological model and especially the production function has a major influence on the convergence speed of the simulated peak discharge distribution towards its asymptote and therefore on its shape for medium range return periods. To illustrate this influence, a second series of computations where conducted with the same rainfall-runoff model including a modified version of the SCS model (see Appendix A1). The conventional SCS model simulates a very progressive convergence of the runoff coefficient towards 1 as the rainfall amount increases, behavior which seems not to be in accordance with some recent observations (Gaume et al., 2004). The proposed modified version of the SCS model simulates a rapid evolution of the runoff coefficient over a given rainfall amount threshold. This behavior induces a change of the convergence speed of the flood peak distribution towards its asymptote (Fig. 6). The flood peak distribution resulting from the DFFD framework has a strange "S" shape with two extremes dominated by the pre and post-threshold behaviors of the rainfall-runoff model and a large transition phase with a much higher slope or curvature than the asymptotic distribution. This is of course a purely theoretical example, but it shows that flood peak distributions may have a large variety of shapes depending on the dynamics of the rainfall-runoff process especially as far as the medium range return periods are concerned. Considering the distance between the peak discharge distributions observed for low return periods and the asymptotic distributions, the range of possibilities is certainly quite large.

\section{Discussion}

\subsection{On the "Gradex" method}

The result concerning the asymptotic behavior of flood peak distributions has some similarities with the "Gradex" theory (Naghettini et al., 1996; Guillot and Duband, 1967), popular in France and in some other countries. This theory states (1) that the distributions of the daily rainfall amount is exponential, (2) that over a given return period value, the mean daily discharge distribution will have the same slope on a semi$\log$ plot than the daily rainfall amount, if both are expressed in the same unit, and (3) that the ratio between mean daily and peak discharges is independent of the return period. But there are some differences.

Firstly, no hypothesis was done here about the type of the asymptotic distribution (EV I or EV II). Secondly, the shape of the peak discharge $Y$ appears linked to the shape of the distribution of $Y_{m}$ which is related to the so called intensity-duration-frequency curves rather than to the shape of the mean daily rainfall amount distribution. Both would be asymptotically equivalent if the ratio between the quantiles of $Y_{m}$ and of the mean daily rainfall amounts were constant, which is generally not the case.

Finally, we have presented here an asymptotic result. The shape of the flood peak distribution and the convergence speed towards its asymptote are highly dependent on the dynamics of the rainfall-runoff process summarized in the density function $p(c \mid x)$. A large variety of FPD shapes can be produced especially if there are thresholds in the rainfallrunoff relation as illustrated herein or previously by Sivapalan et al. (1990). Even if the asymptotic distribution $Y_{m}$ is of the exponential type, its slope on a semi logarithmic paper, which can be assimilated to the Gradex, is not necessarily the maximum possible slope of the flood peak distribution as shown herein. 

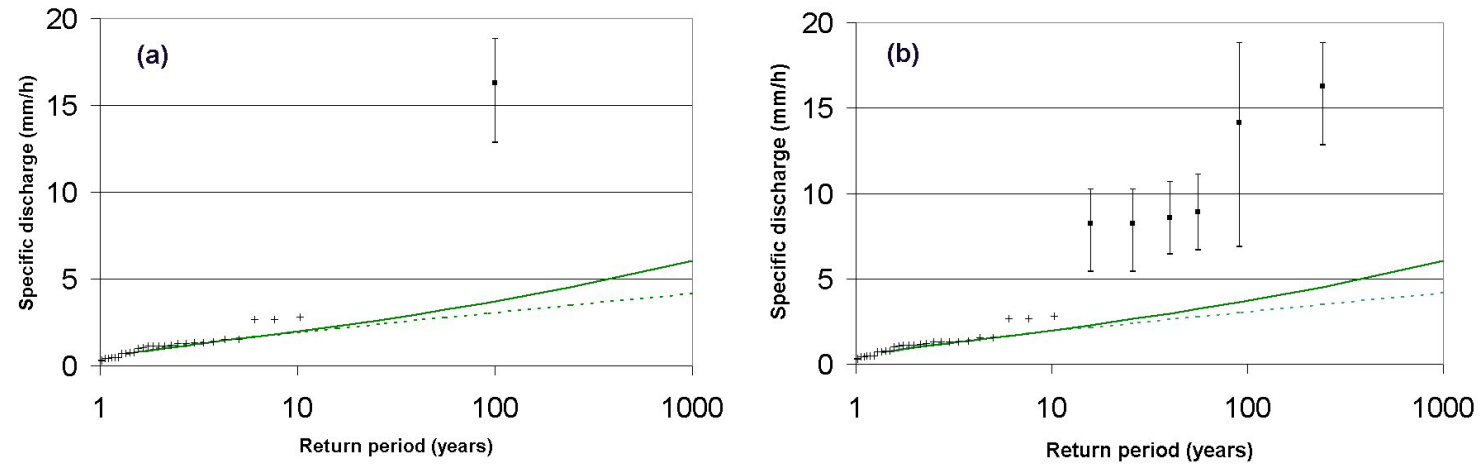

Fig. 7. Empirical flood peak specific discharge distributions of the Clamoux river (French Mediterranean area): (a) based on a series of 24 years of measured data, (b) including the estimated peak discharges of the major floods since 1850 with their estimated ranges of uncertainty. EV I (dotted line) and EV II (continuous line) models calibrated on the 24 years of available measured peak discharges.

\subsection{About statistical extrapolations}

The debate on the estimation of extreme values in hydrology is still lively (Koutsoyiannis, 2004; Klemes, 2000). The results presented herein provide some elements of discussion on this issue. Concerning the quantile estimations of very large return period floods, the distribution of the maximum mean rainfall intensity over a duration of the order of the time of concentration of a watershed should be considered as the possible flood peak asymptotic distribution. Concerning the estimation of medium return period flood quantiles, typically 50 to 500 years, the present paper does not lead to any new proposal. But the awareness of the distance between the asymptotic distribution and the low return period flood quantiles gives an idea of the range of the possibilities for the shape of the flood peak distributions (i.e. for the way the actual distribution will converge towards its asymptote). This is one more argument to take with prudence any quantile estimation uniquely based on statistical extrapolations. Let us illustrate this last idea with the real example of the flood peak distribution of a small river (watershed area of $40 \mathrm{~km}^{2}$ ) located in the south of France (see Fig. 7). This example will also show that the simulated "S" shaped distribution (Fig. 6) is realistic. 24 years of measured discharges are available on the Clamoux river. On 13 November 1999 an extreme flood event occured whose estimated peak specific discharge $(16 \mathrm{~mm} / \mathrm{h})$ lies far over the measured ones. The extrapolation based on the measured series leads to a return period for this discharge of a few hundred thousand years if the EV II distribution is used and of some hundred million years if the EV I distribution is used. But none of these two return periods seem to be in accordance with other information available on the Clamoux river: comparable floods have been observed on the same river during the last century. Moreover, the 100-year maximum mean rainfall intensity over two hours, estimated time of concentration of the Clamoux watershed during the 1999 flood, is about $70 \mathrm{~mm} / \mathrm{h}$ in this area. The estimated peak discharge of the 1999 flood is about four times lower than the maximum possible hundred year quantile. The return period of the 1999 peak specific discharge may therefore be much lower than the value estimated through statistical extrapolations (i.e. of the order of 100 to a few hundred years). A detailed analysis of the data existing on the major historical floods since 1850 (Payrastre et al., 2005) confirms the probably moderate return period of the Clamoux 1999 flood as illustrated on Fig. 7 .

This example is typical for small watersheds in the French Mediterranean area (Payrastre et al., 2005; Gaume et al., 2004). The conclusion is that the "range of the possibilities" for the shape of flood peak distributions is large and, of course, not limited to the theoretical distributions generally used for extrapolation purposes, as for instance the extreme value distributions of type I or II. Thus, how meaningful is it to extrapolate tendencies identified on short series of data for the estimation of larger return-period flood quantiles? As mentioned in the introduction, DFFD tools due to their inherent simplifications, can hardly be considered as an efficient alternative to the conventional statistical extrapolation methods. The uncertainties on estimated flood quantiles can only be really reduced by enlarging the studied data sets using the available information on historical floods as illustrated here and/or combining various data sets in a regional approach. Both, valuation of historical information and regional analysis, should, to our opinion, be systematically part of operational hydrological studies. It is too seldom the case for the moment.

\section{Conclusions}

In summary, it has been shown herein that:

(a) The asymptotic statistical distribution of flood peaks $Y$ obtained through a DFFD approach is of the same type and has the same shape parameter, and scale parameter in the exponential (EV I) case, as the distribution of 
the rainfall mean intensity $X$ for simple DFFD frameworks, i.e. of the maximum possible peak discharge $Y_{m}$ for DFFD accounting for the temporal variations of the intensity during the rainfall event. Of course, $Y, X$ and $Y_{m}$ must have the same units for this result to be valid. In other words, the distribution of $Y$ will appear linear and with the same slope as the distribution of $X$ or $Y_{m}$ on a semi-log plot for exponential type distributions or on a log-log plot for "extreme value" of type II distributions. If the maximum possible value of ratio $C$ between $Y$ and $Y_{m}$ is $c_{2}$ rather than 1 , the same conclusions can be drawn for $Y / c_{2}$.

(b) From a practical point of view the asymptotic properties presented herein shed a new light on previous DFFD results as illustrated in Fig. 3. They also reveal that the distribution of $Y_{m}$ or more simply of the mean maximum rainfall intensity over a duration of the order of the time of concentration of a watershed should be considered and used as a guideline for any extrapolation of a flood peak distribution, especially for large return periods. It should be nevertheless taken into account that the impact of the rainfall spatial heterogeneity has not been considered herein.

(c) Finally, this mainly theoretical work, as other previous works (Bouleau, 1991; Klemes, 2000), puts also in question the common practice of flood quantiles' estimation consisting in extrapolating tendencies observed on generally relatively small measured peak discharge series. It pleads for the systematization of the use of the possible additional information in flood frequencies studies through the valuation of data available on historical floods and regional flood frequency analysis.

\section{Appendix A Presentation of the rainfall-runoff model used in the DFFD numerical simulations}

The rainfall-runoff simulation results presented here have been obtained with a simplified rectangular shaped watershed composed of two rectangular slopes and a central river reach having a rectangular cross-section (see Fig. A1). The main characteristics of the rainfall-runoff model used are as follows: 1) the flood flows are assumed to be essentially composed of surface runoff water, and other sources are set aside, 2) The SCS ("soil conservation service") model is used to calculate the evolution of the mean runoff coefficient on each sub-watershed during the storm event (see Eq. A1), and 3) the flood flows can be either routed through the watershed using a linear transfer function or the "kinematic wave" model. The standard SCS model simulates a progressive and asymptotic growth of the runoff coefficient $C_{t}$ towards 1 as the rainfall amount increases (see Fig. A2).

$C_{t}=1-\left(\frac{S}{\left(P_{t}+0.8 S\right)}\right)^{2}$.

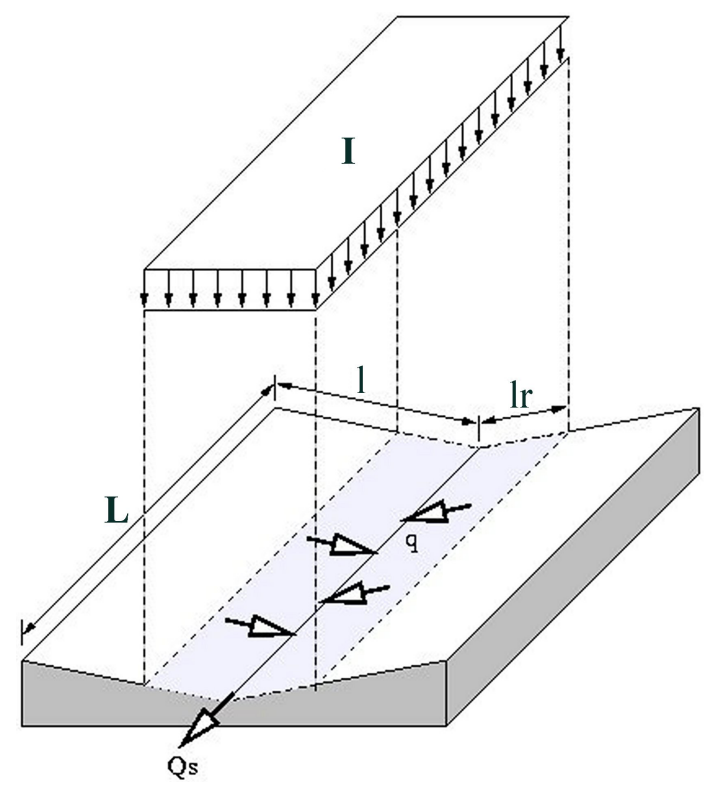

Fig. A1. Representation of a watershed in the rainfall-runoff model.

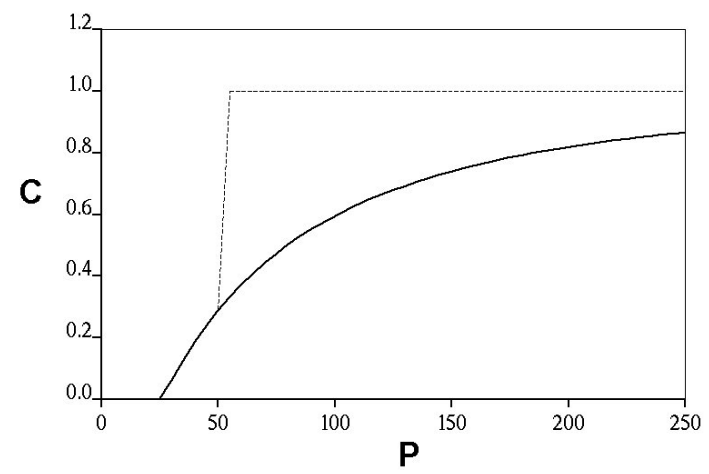

Fig. A2. Relation between the runoff coefficient $C$ and the total rainfall amount $P$ : standard SCS model (bold line) and modified SCS model (dotted line).

This SCS function shape has been selected for mathematical reasons: an asymptotic convergence is the only possible solution for functions including one parameter only. But this asymptotic dynamics does not correspond to some recent hydrological observations (Gaume et al., 2004, 2003). Therefore, an other model including a threshold has been tested: the SCS model is used to compute the runoff rate $C_{t}$ until it reaches $30 \%$ and the runoff rate is set equal to 1 over this threshold (Fig. A2). 
Appendix B Asymptotic distribution of $Y$ when $C$ is independent of $X$ and $X$ is exponentially distributed

Let us begin with the very simple case where $C$ is uniformly distributed over the interval $[0,1]$. The survival function of the flood peak $Y$ then has the following form:

$F(y)=\int_{0}^{1} \int_{y / c}^{\infty} \lambda e^{-\lambda x} d x d c$

or

$F(y)=\int_{0}^{1} e^{-\lambda y / c} d c$.

We are looking for an approximation of this integral when $y$ tends to infinity. One method consists of finding an upper and a lower boundary for this integral that have the same limit when $y$ tends to infinity. Let us first note that $e^{-\lambda y / c}$ is an increasing function of $c$. Therefore, obviously:

$F(y) \leq e^{-\lambda y} \int_{0}^{1} d c$

or

$\log F(y) \leq-\lambda y$.

Moreover, the function $e^{-\lambda y / c}$ takes positive values over the interval $[0,1]$. Then

$F(y) \geq \int_{\epsilon}^{1} e^{-\lambda y / c} d c$

for any $\epsilon$ in $[0,1]$. Recalling that $e^{-\lambda y / c}$ is an increasing function of $c$ :

$F(y) \geq e^{-\lambda y / \epsilon} \int_{\epsilon}^{1} d c$

or

$F(y) \geq e^{-\lambda y / \epsilon}(1-\epsilon)$

or

$\log F(y) \geq-\lambda y\left(\frac{1}{\epsilon}-\frac{\log (1-\epsilon)}{\lambda y}\right)$

for any $\epsilon<1$, as close to 1 as wished, the right hand term of Eq. (B8) tends to $-\lambda y / \epsilon$ when $y$ tends to infinity. We can then write:

$-\lambda y \geq \log F(y) \geq \frac{-\lambda y}{\epsilon}[1+\mathrm{o}(1)]$

where $\mathrm{o}(1)$ stands for a function of $y$ that tends to 0 when $y$ tends to infinity. As $y$ tends to infinity the lower boundary of the inequality (B9) can be taken as close to the upper one as desired. In other words, $\log F(y)$ converges to $-\lambda y$ as $y$ tends to infinity (i.e. the flood peak distribution will asymptotically appear as a straight line with slope $\lambda$ on a conventional semi-logarithmic plot). According to the inequality (B9), $\log F(y)$ has the following asymptotic shape.

$\log F(y)=-\lambda y[1+\mathrm{o}(1)]$.
Equation (B10) is equivalent to

$F(y) \approx h(y) e^{-\lambda y}$

or

$\log F(y) \approx-\lambda y+\log h(y) \approx-\lambda y\left(1-\frac{\log h(y)}{\lambda y}\right)$

with $h(y)$ any function verifying $\lim _{y \rightarrow \infty} \log h(y) /(\lambda y)=0$. In the particular case where $C$ is uniformly distributed over the interval $[0,1]$ and independent of $Y$, it can be shown that $h(y)=1 /(\lambda y)$ (see Appendix C1).

The same demonstration leads to a similar result if $C$ has any distribution independent of $X$ and $Y$ with a strictly positive density over the interval $[0,1]$ :

$F(y)=\int_{0}^{1} p(c) \int_{y / c}^{\infty} \lambda e^{-\lambda x} d x d c$

$F(y)=\int_{0}^{1} p(c) e^{-\lambda y / c} d c$

$F(y)=\int_{0}^{1} e^{-y(\lambda / c+\log [p(c)] / y)} d c$

In this case, whatever the function $p(c)$, the term $\log [p(c)] / y$ tends to 0 when $y$ tends to infinity for any value of $c$. Integrals (B2) and (B15) have the same asymptotic behavior as $y$ tends to infinity.

Let us finally note, to be more general, that the function $p(c)$ can take non-zero values over a reduced interval $\left[c_{1}, c_{2}\right]$ with $0 \leq c_{1} \leq c_{2} \leq 1$. In this case the preceding developments will obviously lead to the following asymptotic relation:

$\log T(y)=\frac{\lambda y}{c_{2}}[1+\mathrm{o}(1)]$

\section{Appendix C Shape of the function $h(x)$ when $C$ is uni- formly distributed over the interval $[0,1]$}

The mathematical developments of this appendix are due to Alain Mailhot of the Institut National de la Recherche Scientifique (Québec, Canada) who suggested them during a discussion about the content of the present paper.

We are looking for an approximation of the following integral as $y$ tends to infinity:

$F(y)=\int_{0}^{1} e^{-\lambda y / c} d c$.

Changing the variable in this integral $u=\lambda y / c$ and $d u=-\lambda y / c^{2} d c$ leads to:

$F(y)=-\lambda y \int_{+\infty}^{\lambda y} \frac{e^{-u}}{u^{2}} d u$. 
This integral can be evaluated using integration by parts:

$F(y)=-\lambda y\left[\frac{e^{-u}}{u}\right]_{+\infty}^{\lambda y}+\lambda y \int_{+\infty}^{\lambda y} \frac{e^{-u}}{u} d u$

or

$F(y)=e^{-\lambda y}-\lambda y \int_{\lambda y}^{+\infty} \frac{e^{-u}}{u} d u$.

The second term of Eq. (C4) has a well known Taylor expansion:

$F(y)=e^{-\lambda y}-\lambda y \frac{e^{-\lambda y}}{\lambda y}\left(1-\frac{1 !}{\lambda y}+\frac{2 !}{(\lambda y)^{2}}-\ldots\right)$

so

$F(y)=\frac{e^{-\lambda y}}{\lambda y}\left(1-\frac{2 !}{\lambda y}+\frac{3 !}{(\lambda y)^{2}}-\ldots\right)$.

We can then conclude that when $y$ tends to infinity:

$F(y) \approx \frac{e^{-\lambda y}}{\lambda y}$.

\section{Appendix D Asymptotic distribution of $Y$ when $C$ is in- dependent of $X$ and the statistical distribution of $X$ is an extreme value distribution of type II (Fréchet)}

The density function of the rainfall intensities has the following form:

$p(x)=\frac{\alpha}{b}\left(\frac{x-a}{b}\right)^{-(\alpha+1)} e^{-\left(\frac{x-a}{b}\right)^{-\alpha}}$

with $\alpha>0$. If $p(c)$ is the density function of the runoff rate $C$, the survival function of the peak discharges is:

$P(Y>y)=F(y) \int_{0}^{1} p(c)\left(1-e^{-\left(\frac{y / c-a}{b}\right)^{-\alpha}}\right) d c$.

The term $e^{-\left(\frac{y / c-a}{b}\right)^{-\alpha}}$ tends to $1-\left(\frac{y / c-a}{b}\right)^{-\alpha}$ when $y$ tends to infinity. Then

$F(y) \approx \int_{0}^{1} p(c)\left(\frac{y / c-a}{b}\right)^{-\alpha} d c$.

This integral can be furthermore simplified when $y$ tends to infinity:

$F(y) \approx \int_{0}^{1} p(c)\left(\frac{y}{c b}\right)^{-\alpha} d c$

or

$F(y) \approx \int_{0}^{1} p(c) c^{\alpha}\left(\frac{y}{b}\right)^{-\alpha} d c$

which is equivalent to

$F(y) \approx\left(\frac{y}{b}\right)^{-\alpha} \int_{0}^{1} p(c) c^{\alpha} d c$. $\alpha$ is greater than 0 by definition of the EV II distribution and $p(c)$ is the density function of a variable defined over the interval $[0,1]$ :

$\int_{0}^{1} p(c) d c=1$.

Necessarily

$0 \leq \int_{0}^{1} p(c) c^{\alpha} d c \leq 1$.

Let $A$ be the limit of this integral when $y$ tends to infinity, $A \in[0,1]:$

$A=\lim _{y \rightarrow \infty}\left(\int_{0}^{1} p(c) c^{\alpha} d c\right)$.

The result is much more simple than the one obtained in the exponential case. Unless $A$ is equal to zero, which is nonrealistic for a rainfall-runoff model since it implicates that $c$ tends to zero when $y$ tends to infinity, the asymptote of the survival function of $Y$ is a EV II function with the same shape parameters $\alpha$ as the rainfall intensity survival function.

$F(y) \approx A\left(\frac{y}{b}\right)^{-\alpha}$

(D10)

Acknowledgements. The author thanks Alain Mailhot for the motivating discussions about the content of this paper and his contribution (see Appendix C1) as well as Demetris Koutsoyiannis who suggested the demonstration based on the moments of the distributions presented in the first part of the paper and contributed to a significant improvement of the manuscript.

Edited by: L. G. Lanza

\section{References}

Arnaud, P. and Lavabre, J.: Using a stochastic model for generating hourly hyetographs to study extreme rainfalls, Hydrol. Sci. J., 44, 433-446, 1999.

Arnaud, P. and Lavabre, J.: La modélisation stochastique des pluies horaires et leur transformation en débits pour la prédétermination des crues, Revue et Sciences de l'Eau, 13, 441-462, 2000.

Arnaud, P. and Lavabre, J.: Coupled rainfall model and discharge model for flood frequency estimation, Water Resour. Res., 38 , 1075, doi:10.1029/2001WR000474, 2002.

Blazkova, S. and Beven, K.: Flood frequency estimation by continuous simulation of a catchment treated as ungauged (with uncertainty), Water Resour. Res., 38, 1139, doi:10.1029/2001WR000500, 2002.

Blazkova, S. and Beven, K.: Flood frequency estimation by continuous simulation of subcatchment rainfalls and discharges with the aim of improving dam safety assessment in a large basin in the Czech Republic, J. Hydrol., 292, 153-172, 2004.

Bouleau, N.: Splendeurs et misères des lois des valeurs extrêmes, Risques, 3, 85-92, 1991. 
Cameron, D., Beven, K., and Naden, P.: Flood frequency estimation by continuous simulation under climate change (with uncertainty), Hydrol. Earth Syst. Sci., 4, 393-405, 2000.

De Michele, C. and Salvadori, G.: On the derived flood frequency distribution : analytical formulation and the influence of the antecendent soil moisture condition, J. Hydrol., 262, 245-258, 2002.

De Michele, C. and Salvadori, G.: A generalized Pareto intensityduration model of storm rainfall exploiting 2-Copulas, J. Geophys. Res., 108, 4067, doi:10.1029/2002JD002534, 2003.

Diaz-Granados, M., Valdes, J., and Bras, R.: A physical based flood frequency distribution, Water Resour. Res., 20, 995-1002, 1984.

Eagleson, P.: Dynamics of flood frequency, Water Resour. Res., 14, 878-898, 1972.

Gaume, E., Livet, M., and Desbordes, M.: Study of the hydrological processes during the Avene river extraordinary flood (south of France): 6-7 October 1997, Phys. Chem. Earth, 28, 263-267, 2003.

Gaume, E., Livet, M., Desbordes, M., and Villeneuve, J.-P.: Hydrological analysis of the river Aude, France, flash flood on 12 and 13 November 1999, J. Hydrol., 286, 135-154, 2004.

Goel, N., Kurothe, R., Mathur, B., and Vogel, R.: A derived flood frequency distribution for correlated rainfall intensity and duration, J. Hydrol., 228, 56-67, 2000.

Guillot, P. and Duband, D.: La méthode du GRADEX pour le calcul de probabilités des crues à partir des pluies, IAHS Publication, 84, 1967.

Gupta, V., Castro, S., and Over, T.: On scaling exponents of spatial peak flows from rainfall and river network geometry, J. Hydrol., 187, 81-104, 1996.

Hashemi, A., Franchini, M., and O'Connell, P.: Climatic and basin factors affecting the flood frequency curve: Part I - A simple sensitivity analysis based on the continuous simulation approach, Hydrol. Earth Syst. Sci., 4, 463-482, 2000.
Iacobellis, V. and Fiorentino, M.: Derived distribution of floods based on the concept of partial area coverage with a climatic appeal, Water Resour. Res., 36, 469-482, 2000.

Klemes, V.: Tall tales about tails of hydrological distributions, I and II, J. Hydrol. Eng., 5, 225-239, 2000.

Koutsoyiannis, D.: Statistics of extremes and estimation of extreme rainfall: I.Theoretical investigation, Hydrol. Sci. J., 49, 575-590, 2004.

Lamb, R. and Kay, A.: Confidence intervals for a spatially generalised continuous simulation flood frequency model for Great Britain, Water Resour. Res., W07501, 40, doi:10.1029/2003WR002776, 2004.

Loukas, A.: Flood frequency estimation by a derived distribution procedure, J. Hydrol., 255, 69-89, 2002.

Moughamian, M., McLaughlin, D., and Bras, R.: Estimation of flood frequency : an evaluation of two derived distribution procedures, Water Resour. Res., 23, 1309-1319, 1987.

Mouhous, N., Gaume, E., and Andrieu, H.: Influence of the highest values on the choice of log-poisson random cascade model parameters, Phys. Chem. Earth, 26, 701-704, 2001.

Naghettini, M., Potter, K., and Illangasekare, T.: Estimating the upper tail of flood-peak frequency distributions using hydrometeorological information, Water Resour. Res., 32, 1729-1740, 1996.

Payrastre, O., Gaume, E., and Andrieu, H.: Use of historical data to assess the occurrence of floods in small watersheds in the French mediterranean area, Adv. Geosci., 2, 313-320, 2005.

Raines, T. and Valdes, J.: Estimation of flood frequency for ungaged catchments, J. Hydraul. Eng., 119, 1138-1154, 1993.

Sivapalan, M., Wood, E., and Beven, K.: On hydrologic similarity 3. A dimensionless flood frequency model using a generalized geomorphologic unit hydrograph and partial area runoff generation, Water Resour. Res., 26, 43-58, 1990.

Smith, J.: Representation of basin scale in flood peak distributions, Water Resour. Res., 28, 2993-2999, 1992. 